\title{
An Adaptive Energy Aware Clustering Based Reliable Routing for in-Network Aggregation in Wireless Sensor Network
}

\author{
A. Manickavasuki \\ Cape Institute Of Technology \\ Levengipuram, India
}

\author{
R. Ramya \\ Cape Institute Of Technology \\ Levengipuram,India
}

\begin{abstract}
Wireless Sensor Network (WSN) consists of spatially distributed autonomous devices that cooperatively sense physical or environmental conditions. Due to the non-uniform node deployment, the energy consumption among nodes are more imbalanced in cluster-based wireless sensor networks this factor will affect the network life time. Cluster-based routing and EADC algorithm through an efficient energy aware clustering algorithm is employed to avoid imbalance network distribution. Our proposed protocol EADC aims at minimizing the overall network overhead and energy expenditure associated with the multi hop data retrieval process while also ensuring balanced energy consumption among SNs and prolonged network life time .A optimal one-hop based selective node in building cluster structures consisted of member nodes that route their measured data to their assigned cluster head is identified to ensure efficient communication. The proposed routing algorithm increases forwarding tasks of the nodes in scarcely covered areas by forcing cluster heads to choose nodes with higher energy and fewer member nodes and finally, achieves imbalanced among cluster head and improve the network life time.
\end{abstract}

Keywords: Cluster Head(CH),Sink,EADC

\section{INTRODUCTION}

Sensor nodes measure various parameters of the environment and transmit data collected to one or more sinks using hop-byhop communication. The main goal of WSN is to collect useful information as much as possible in the monitoring area, which implies that energy efficiency and lifetime of networks are very important. In wireless sensor networks, sensor nodes are usually powered by batteries that cannot be replaced in most cases. As a result, the energy constraint has significant effect on the network design and makes energy efficiency a major design challenge. In order to achieve high energy efficiency and increase network lifetime, sensors are often hierarchically organized into clusters.

Typically, a clustering algorithm divides the network into subsets of nodes, called clusters, each with one serving as the as the cluster head. Within a cluster, each node has its own cluster head $(\mathrm{CH})$ and transmits data to its $\mathrm{CH}$ over relatively short distance, which in turn forwards the data to the sink.

Cluster heads aggregate the data from their cluster members, and send the aggregated data to the sink. Communications between cluster heads and the BS is single-hop communication and multi-hop communication. In single-hop communication the data packets are directly send to the $\mathrm{CH}$ or $\mathrm{BS}$ without any relay node. So If any $\mathrm{CH}$ node will be die due to some power failure and data will be lost. To overcome this using multi-hop communication clustering algorithms, the energy consumption of cluster heads consists of the energy for receiving, aggregating and sending the data from their cluster members (intra-cluster energy consumption) and the energy for forwarding data for their neighbor cluster heads (intercluster energy consumption).
In previous method DRINA paper is used on the routing techniques in uniform node distribution in clustering based techniques. But this approach is not suitable in the non uniform node distribution and load balancing in sensor network. Imbalance in network traffic load has a negative effect on network lifetime since transmit and receive operations are not evenly spread among network nodes.

In this paper, we propose a cluster-based routing protocol for wireless sensor networks with non uniform node distribution whose cores are an energy-aware clustering algorithm EADC and a cluster-based routing algorithm. EADC constructs clusters of even sizes using competition range in order to balance the energy consumption among cluster members. To solve the imbalanced energy consumption among cluster heads caused by the non uniform node distribution, a clusterbased routing algorithm is used for balance the energy consumption among cluster heads by adjusting the intracluster and inter-cluster energy consumption of cluster heads. Therefore, it can achieve the balance of energy consumption among nodes and prolong the network lifetime.

\section{RELATED WORK}

Cluster routing is an energy efficient routing model as compared with direct routing and Multihop routing A new adaptive cluster routing algorithm CIDRSN (Cluster ID based Routing in Sensor Networks) Cluster routing is adopted in this research work. In this research work Cluster ID based routing is adopted and adaptive cluster size is proposed.

In this algorithm Cluster ID is used as next hop rather than $\mathrm{CH}-\mathrm{ID}$ in routing table. In this way cluster formation process is eliminated for each round. Cluster formation is only carried out in start thus reduces the energy consumption and increases 
the network lifetime to about $16 \%$ A maximum-Votes and Load-balance Clustering Algorithm (VLCA) was presented by (Zhang et al., 2008) to reduce the number of clusters and prolong network lifetime. To balance the workload among cluster-heads, this algorithm selects the cluster-head by considering the number of member nodes and the residual battery level. (Murthy et al., 2008) proposed a level controlled clustering to reduce the number of messages toward the base station and increase the network lifetime of WSN. This method assumes that the base station is able to transmit at various power levels. The cluster head selection method is also based on the maximum residual battery level.

PEACH (Power Efficient And Adaptive Clustering Hierarchy) is a cluster formation technique based on overheard information from the sensor nodes. According to this approach, if a cluster head node becomes an intermediate node of a transmission, it first sets the sink node as its next hop. Then it sets a timer to receive and aggregate multiple packets from the nodes in the cluster set for a pre-specified time. It checks whether the distance between this node and the original destination node is shorter than that between this node and the already selected next hop node. If the distance is shorter, this node joins to the cluster of the original destination node and the next hop of this node is changed to the original destination node. PEACH is an adaptive clustering approach for multi-hop inter-cluster communication. How-ever, it suffers from almost the same limitations of PEGASIS due to the choice of physical propinquity.

Clustering Network Topology Control the CNTCABRT algorithm follows the same clustering principle as the LEACH algorithm. Studies on the mechanism of wireless sensor network data transmission have revealed that sensor network data transmission improves with responsibility transmission. Thus, we pro- posed an algorithm that selects the cluster head through the strategy of accumulating evidence with responsibility. Briefly, the algorithm periodically divides the whole sensor network into several clusters, with each cycle referred to as a round. Each round involves cluster formation and data transmission. The algorithm exhibited good performance in controlling cluster head selection, cluster deployment, and cluster size.

All the algorithms mentioned above are all based on the assumption that all the nodes are uniformly distributed in the networks. In networks with non uniform node distribution, considering the network coverage problem, proposed some good cluster head election techniques. However, this paper focused on coverage preservation, while the energy consumption balance and network lifetime on the back burner. Considering the node distribution, the authors in proposed a hierarchical architecture of sensor network with cluster formation and cluster head selection algorithm. The authors used various parameter metrics related to node density and indicate the deployment density variation of nodes by the edge of link lengths standard deviations. In proposed an energy-aware distributed unequal clustering protocol
(EADUC) in multi-hop heterogeneous wireless sensor networks to "energy hole" problem

\section{NETWORK MODEL}

A network system consists of static and battery-powered sensor nodes as well as one sink node. Sensor nodes are distributed randomly in a reliable environment and have the same physical capabilities. . The sink node is responsible for receiving the user query, propagating it through a like tree structure, and then collecting the results from the sensors. All the nodes and the BS are stationary after deployment. All the sensor nodes can be heterogeneous, but whose energy cannot be recharged. All the sensor nodes are location-unaware. All the nodes can use power control to vary the amount of transmit power. The BS is out of the sensor field. It has a sufficient energy resource and the location of the BS is known by each node. cluster heads to choose nodes with higher energy and fewer member nodes as their next hops, and finally, achieves load balance among cluster heads.

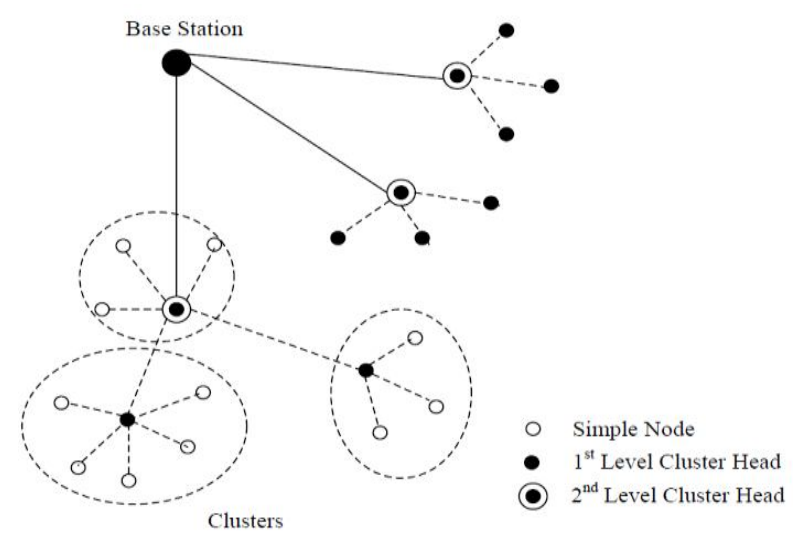

Fig 1:Network Model

\subsection{Cluster based Routing Algorithm}

This protocol contains an energy-aware clustering algorithm EADC and a cluster-based routing algorithm. In order to elect cluster heads with higher energy, the parameter of cluster head competition in EADC is based on the ratio between the average residual energy of neighbor nodes and the residual energy of the node itself. Moreover, cluster heads broadcast head messages using the same competition range to construct clusters of even sizes. Thus, the energy consumption among cluster members can be balanced well. However, the even cluster size also makes the energy consumption among cluster heads imbalanced, due to non uniform distribution of nodes. Cluster heads in dense areas have more members nodes, so they have high intra-cluster energy consumption. For this, we propose an inter-cluster energy-efficient multi-hop routing protocol, in which cluster heads select the neighbor cluster head with higher residual energy and a smaller number of cluster members as the next hop to balance the energy consumption among cluster heads. 


\section{EADC DETAILS}

This process is similar to the cluster set-up phase in EADC proposed previously by us. The whole process is divided into three phases: information collection phase, whose duration is $\mathrm{T} 1$; cluster head competition phase, whose duration is $\mathrm{T} 2$; cluster formation phase, whose duration is $\mathrm{T} 3$.

\subsection{Information collection phase}

The duration of the phase is defined as T1, during which each node broadcasts a Node Msg with the following two values: one is the node id, and the other is the residual energy of this node within radio range $r$. At the same time, it receives the Node messages from its neighbor nodes, according to which, e each node si calculates the average residual energy.

\subsection{Cluster Head Selection Phase}

When $\mathrm{T} 1$ has expired, EADC begins the cluster head competition phase whose duration is T2. In this phase, if node si receives no Head Msg when timer ti expires, it broadcasts the Head Msg within radio range Rc to advertise that it will be a cluster head. Otherwise, it gives up the competition. The following pseudo-code gives the details of this phase.

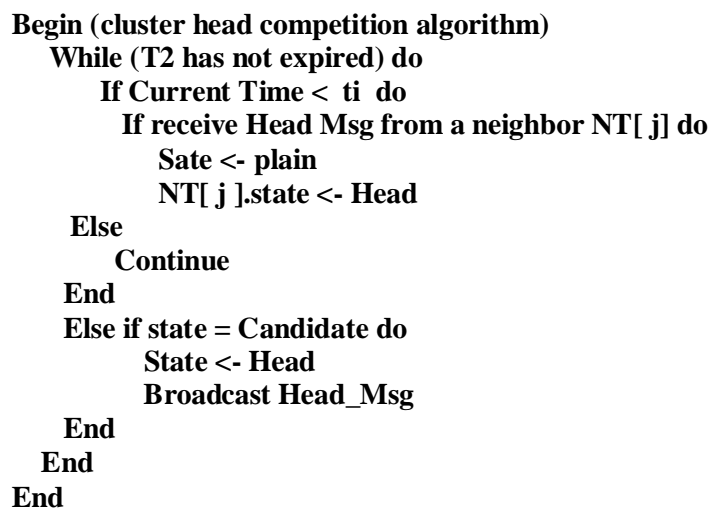

\subsection{Cluster Formation Phase}

After T2 expires, the last phase of EADC is the cluster formation phase, we define the duration as T3. In this phase, each non-cluster head node chooses the nearest cluster head and sends the Join Message which contains the id and residual energy of this node. According to the received Join Messages, each cluster head creates a node schedule list including the Schedule Message for its cluster members, the Schedule Message is used for telling the cluster members when they can transmit their data to the cluster head and in other time interval they can alter their state to asleep to reduce the energy consumption. At this point, the entire process of EADC is completed. Each cluster is composed of the nodes in the Voronoi cell around the cluster head.

\subsection{Routing Formation}

The elected group leader, starts establishing the new route for the event dissemination. For that, the cluster head sends a route establishment message to its NextHop node. When the NextHop node receives a route establishment message, it retransmits the message to its NextHop and starts the hop tree updating process. These steps are repeated until either the sink is reached or a node that is part of an already established route is found. The routes are created by choosing the best neighbor at each hop. The choices for the best neighbor are twofold:

1) When the first event occurs, the node that leads to the shortest path to the sink is chosen and

2) After the occurrence of subsequent events, the best neighbor is the one that leads to the closest node that is already part of an established route .This process tends to increase the aggregation points, ensuring that they occur as close as possible to the events. The resulting route is a tree that connects the Coordinator nodes to the sink. When the route is established, the hop tree updating phase is started. The main goal of this phase is to update the HopToTree value of all nodes so they can take into consideration the newly established route. This is done by the new relay nodes that are part of an established route. These nodes send an HCM message (by means of a controlled flooding) for the hop updating. The whole cost of this process is the same of a flooding, i.e., each node will send only one packet.

\subsection{Performance Analysis}

The lifetime of a WSN can be quantified using the following three kinds of metrics: (1) the time from the deployment of the network to the death of the first node.

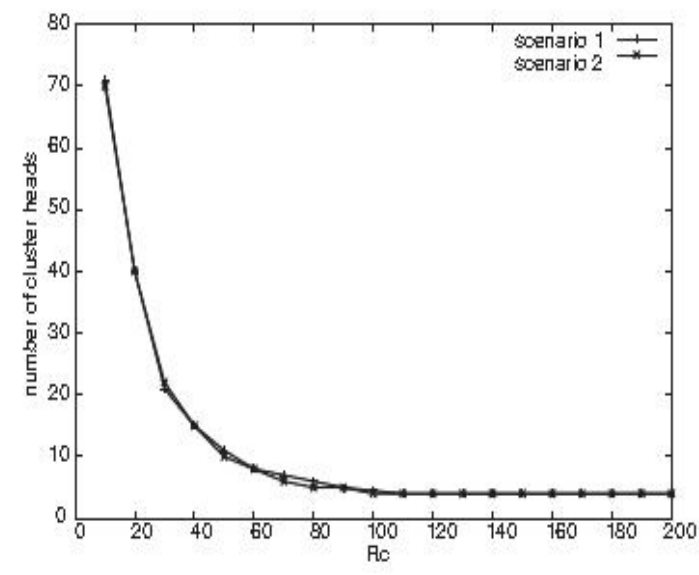

Fig. 2 Number of cluster heads generated in each scenario.

(2) the time when a certain percent of nodes alive (3) the time when all the nodes are dead in the network. The network lifetime is defined as the time when 90 percent of nodes alive.

In Fig. 2 it exhibits the number of cluster heads generated in each scenario. As shown in the figure, the two curves coincide roughly which means that the number of cluster generated in the two scenarios are approximately equal. The reason for this phenomenon is that Rc controls the coverage of cluster head, so that clusters have uniform cluster sizes. Therefore, the number of cluster heads is unaffected by the node distribution. In addition, the uniform cluster size ensures the balance energy consumption among cluster members. 
In wireless sensor network, too many or too few of the cluster heads will cause energy waste, affecting the network lifetime fig. 3 the number of cluster heads decreases when Rc increases.

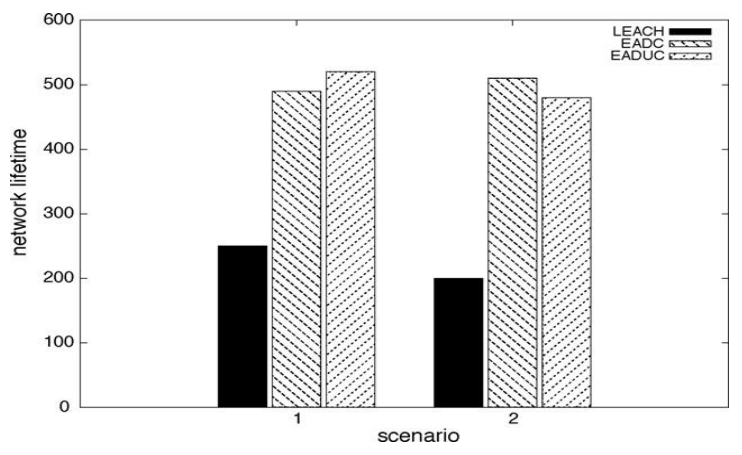

Fig. 3 the number of cluster heads decreases when Rc increases.

Furthermore, the rates of decrease gradually slow down. Therefore, the network lifetime should increase gradually with the increase of Rc. In EADC performance is better than LEACH in prolonging network lifetime. The reason is that LEACH does not take into account the energy of nodes while choosing cluster heads.fig 4 increase the throughput of the system and EADC overcomes the imbalance energy consumption problem by introducing a cluster based intercluster routing protocol. EDAC can solve the non uniform distribution and heterogeneity of nodes well, and prolong the network lifetime significantly.

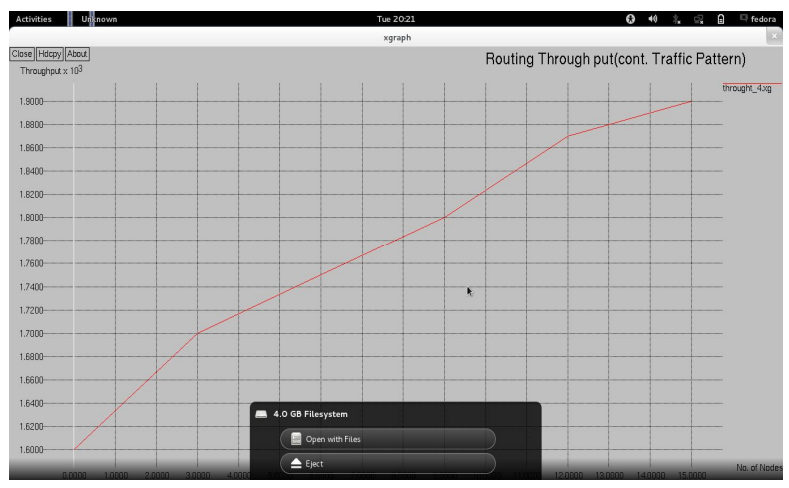

Fig. 4 Throughput Performance

From fig. 4 we can see that EADUC and EADC perform far between than LEACH in prolonging network lifetime. The reason is that LEACH does not take into account the energy of nodes while choosing cluster heads. In our system, the low energy nodes restrict the network lifetime, and the energy of high-energy nodes is wasted.

\section{CONCLUSION}

In this paper, we propose a cluster-based routing protocol for wireless sensor networks with non uniform node distribution which contains an energy-aware clustering algorithm and a cluster based routing algorithm. The clustering algorithm balances the energy consumption among cluster members by constructing equal clusters. Inevitably, the energy consumption among cluster heads is imbalance due to the non uniform node distribution. Therefore, we propose a clusterbased inter-cluster routing algorithm to balance the energy consumption among cluster heads by adjusting intra-cluster energy consumption and inter-cluster energy consumption. Each cluster head chooses a cluster head with higher residual energy and fewer cluster members as its next hop. The imbalanced energy consumption caused by non uniform node distribution is solved by increasing forwarding task of the cluster heads in sparse areas. By using the above mechanisms, our protocol can take advantage of the non uniform distribution and heterogeneity of nodes well, and prolong the network lifetime significantly.

\section{REFERENCES}

[1] S. Olariu, Q. Xu, and A. Zomaya, "An EnergyEfficient Self- Organization Protocol for Wireless Sensor Networks," Proc. IEEE Intelligent Sensors, Sensor Networks and Information Processing Conf. (ISSNIP), pp. 55-60, Dec. 2004

[2] B. Krishnamachari, D. Estrin, and S.B. Wicker, "The Impact of Data Aggregation in Wireless Sensor Networks," Proc. 22nd Int'l Conf. Distributed Computing Systems (ICDCSW '02), pp. 575-578, 2002.

[3] H.S. AbdelSalam and S. Olariu, "A Lightweight Skeleton Construction Algorithm for Self-Organizing Sensor Networks," Proc. IEEE Int'1 Conf. Comm. (ICC), pp. 1-5, http://dblp. uni-trier. $\mathrm{de} / \mathrm{db} / \mathrm{conf} / \mathrm{icc} / \mathrm{icc} 2009 . \mathrm{html}$ \#AbdelSalamO09

[4] C. Intanagonwiwat, D. Estrin, R. Govindan, and J. Heidemann, "Impact of Network Density on Data Aggregation in Wireless Sensor Networks," Proc. 22nd Int'l Conf. Distributed Computing Systems, pp. 457-458, 2002.

[5] E.F. Nakamura, H.A.B.F. de Oliveira, L.F. Pontello, and A.A.F. Loureiro, "On Demand Role Assignment for Event-Detection in Sensor Networks," Proc. IEEE 11th Symp. Computers and Comm. (ISCC '06), pp. 941-947, 2006.

[6] S. Madden, M.J. Franklin, J.M. Hellerstein, and W. Hong, "Tag: A Tiny Aggregation Service for Ad-Hoc Sensor Networks," ACM SIGOPS Operating Systems Rev., vol. 36, no. SI, pp. 131-146, 2002.

[7] S. Madden, R. Szewczyk, M.J. Franklin, and D. Culler, "Support- ing Aggregate Queries over Ad-Hoc Wireless Sensor Networks," Proc. IEEE Fourth Workshop Mobile Computing Systems and Applica- tions (WMCSA '02), pp. 49-58, 2002.

[8] A.P. Chandrakasan, A.C. Smith, and W.B. Heinzelman, "An Application-Specific Protocol Architecture for Wireless Microsen- sor Networks," IEEE Trans. Wireless Comm., vol. 1, no. 4, pp. 660- 670, Oct. 2002.

[9] L.A. Villas, A. Boukerche, R.B. Araujo, and A.A. Loureiro, "A Reliable and Data Aggregation Aware Routing Protocol for Wireless Sensor Networks," Proc. 12th ACM Int'l Conf. Modeling, Analysis and Simulation of Wireless and Mobile Systems (MSWiM), 